\title{
Minimizing recalibration using a non-linear regression technique for thermal anemometry
}

\author{
Rishav Agrawal ${ }^{1} \cdot$ Richard D. Whalley $^{2} \cdot$ Henry C.-H. Ng$^{1}{ }^{10} \cdot$ David J. C. Dennis $^{1} \cdot$ Robert J. Poole $^{1}$
}

Received: 22 February 2019 / Revised: 3 May 2019 / Accepted: 4 June 2019 / Published online: 1 July 2019

(c) The Author(s) 2019

\begin{abstract}
A new method to minimize recalibration in thermal anemometry using a non-linear regression technique is investigated. This method finds potential applications in cases of correcting for non-thermal calibration drifts in long measurements and scenarios where direct calibration of the hot films/hot wires is not possible or suffers significant uncertainties. The essential input for this technique is the a priori knowledge of the first three or four moments of velocity or wall-shear stress for a given Reynolds number. These can be obtained from a separate database (experimental or numerical) in cases where this technique is used as an alternative to direct calibration, or from a previous or simultaneous set of experiments when correcting for non-thermal calibration drifts. Using this input, the coefficients for the assumed calibration functional form can be obtained by an error minimization process. Illustrative results are shown for channel flows where glue-on hot-film probes and hotwire probes are used for wall-shear stress and streamwise velocity measurements, respectively. There is found to be a good agreement between the velocity and wall-shear stress obtained using regression and prior calibration, which is confirmed using both time history and probability density function plots. Sensitivity to the form of calibration relationship, number of moments, and number of samples required for the regression are conducted. Through examples, it is observed that this method works well in estimating the data when moments obtained from a numerical database are used and also works well in correcting for non-thermal calibration drifts. This technique is also shown to work well for the estimation of data from the voltage signals if moments are available for a Reynolds number "close" to, but not the same as, the measured Reynolds number. One additional potential scenario for application related to the measurement in external flows is then discussed.
\end{abstract}

Henry C.-H. Ng

hchng@liverpool.ac.uk

1 School of Engineering, University of Liverpool, Liverpool L69 3GH, UK

2 School of Engineering, Newcastle University, Newcastle NE1 7RU, UK 


\section{Graphic abstract}

\section{Non-linear regression technique}

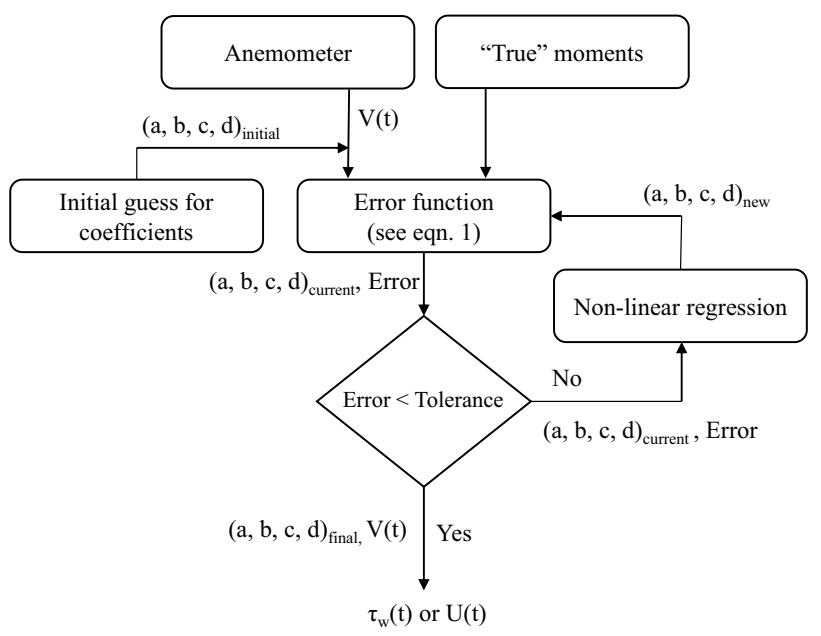

Application to non-thermal drift correction

Probe 1, probe 2

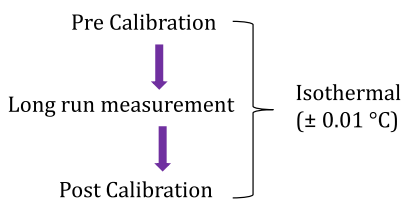

$$
\begin{array}{cc}
\text { Probe 1: Pre Calibration }=\text { Post Calibration } \\
\text { (No drift) }
\end{array} \quad \begin{gathered}
\text { Use moments obtained } \\
\text { from probe } 1 \text { to conduct } \\
\text { non-linear regression on } \\
\text { voltage data from probe 2 }
\end{gathered}
$$

(Non-thermal drift)

Illustrative plots
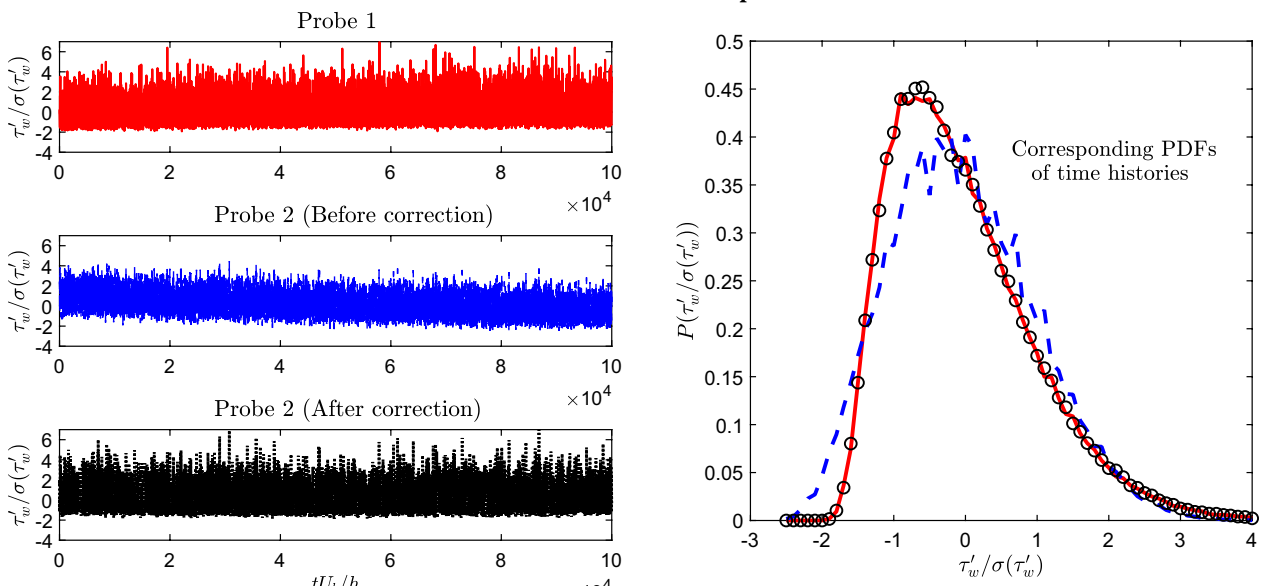

\section{Introduction}

The use of constant temperature anemometry (CTA) for velocity and wall-shear stress measurements in turbulent flows is well documented in the literature. The fundamental principle of this technique is to keep the hot-wire/hot-film sensor at a constant mean temperature where the voltage change to do this is the measure of local velocity or wall-shear stress fluctuations 
(Bruun 1995). Two of the common challenges in this measurement technique are calibration against a known mean value and keeping the calibration drift to a minimum for the entire measurement run. As for the first challenge, many methods have been employed over the past decades for practical and accurate calibration of hot-wire/hot-film probes. For hot-wire measurements in air flows, the hot wire is calibrated against the mean velocity measured in a known flow. The hot wire can be calibrated with a purpose built calibration device or, in situ where, for example, a hot wire may be calibrated in the free stream of a wind tunnel against the mean velocity measured by a Pitot-Static tube pair (e.g., Hutchins et al. 2009). Calibration of hot-film wall-shear stress sensors is also commonly conducted against the mean measured pressure drop along the streamwise direction for fully developed channel (e.g., Whalley et al. 2017) and pipe flows. Although these calibration procedures work well in providing the respective time series of velocities or wall-shear stresses, there are still many examples where the calibration of sensors is either inaccurate or quite challenging. For example, in many external flows (e.g., turbulent boundary layer), correlations such as the Clauser chart are required to calibrate hot films (Hutchins et al. 2011). As stated before, another commonly encountered challenge in thermal anemometry is to keep the calibration constant for the entire measurement run. The calibration drifts which are commonly observed can be attributed to temperature change (Comte-Bellot 1976), commonly referred to as thermal drift, or non-thermal drifts which can be caused by various reasons such as contaminant deposition on the sensing element (see, for example Collis 1954, who studied the effect of dust on hot wires). Correction of thermal drifts has been investigated using various techniques in the past (see, for example: Cimbala and Park 1990; Bruun 1995; Tropea et al. 2007; Hultmark and Smits 2010). However, only a few works have been done to correct for non-thermal calibration drifts in thermal anemometry (Durst et al. 1996; Talluru et al. 2014). The present work attempts to address the issues related to the challenges in calibrations and correcting for non-thermal calibration drifts by investigating a novel technique which is based on non-linear regression. The inputs for this technique are the first three or four moments of the velocity or wall-shear stress for the corresponding Reynolds number. The voltage signals obtained using either a hot wire or hot film for the same flow conditions can then be converted to the resultant time series of velocity or wall-shear stress, respectively, via an error minimization process that has nominally equivalent moments as the given input. Here, this technique is shown to work well in recovering the time series for the wall-shear stress data obtained using glueon hot films and streamwise velocity data obtained using hot wires. It will be shown that the data from a sensor which has suffered non-thermal drifts can be corrected by this technique, thus opening a potential for practical applications. An attempt is also made to estimate the time history of wall-shear stress using the moments obtained from a direct numerical simulation (DNS) database and a good agreement is observed. Thus, this technique also has an opportunity to solve the challenges related to calibration in various flows where a direct calibration is impractical or not possible.

\section{Experimental set-up}

Experiments are conducted in a channel flow facility having an aspect ratio [half-width $(w)$ /half-height $(h)$ ] of 11.92 , where a water-glycerine mixture (of varying concentration) is used as the working fluid. Pressure-drop measurements are conducted using a Druck LPX-3981 differential pressure transducer which has a working range of $5 \mathrm{kPa}$ and an accuracy of $\pm 5 \mathrm{~Pa}$. Hot-film anemometry is employed to measure instantaneous wall-shear stress where a Dantec 55R48 glue-on hot-film probe is used as the sensor. The probe is powered by a Dantec StreamLine Pro velocimetry system and is operated under constant temperature (CT) mode. The probe is located $5 h$ away from the side wall $(z / h=5$, where $z$ is the distance from the side wall) and 496 channel half-heights away from the inlet $(x / h=496$, where $x$ is the streamwise distance). The spanwise width of the hot-film sensing element for $R e_{\tau}=h u_{\tau} / v=180$ is $l^{+}=l u_{\tau} / v=13\left(l^{+} \lesssim 20-25\right.$ is considered acceptable for well-resolved turbulence measurements, see: Ligrani and Bradshaw 1987) where $l$ is the spanwise width of the sensor, $u_{\tau}$ is the friction velocity, and $v$ is the kinematic viscosity. Hot-film calibration is conducted against pressuredrop measurements made by the pressure transducer in the fully developed region of the channel flow. To avoid any thermal drift in the output voltage, the temperature of the working fluid is controlled to a precision of $\pm 0.01{ }^{\circ} \mathrm{C}$ for the entire experimental run using a heat exchanger. The results shown here are for three friction Reynolds numbers, $R e_{\tau}=61,84$, and 180. Figure 1 shows a typical calibration plot for the hot film obtained at $R e_{\tau}=180$. Two commonly used calibration equations: third-order polynomial $\left(V=a_{1} q^{3}+b_{1} q^{2}+c_{1} q+d_{1}\right)$ and extended power law $\left(V^{2}=a_{2} q^{2 d_{2}}+b_{2} q^{d_{2}}+c_{2}\right)(\mathrm{Wu}$ and Bose 1994) are used here to fit the data, where $q$ represents the physical quantity (wall-shear stress or velocity) and $V$ represents the mean output voltage. It is observed that both types of fit work well in fitting the calibration points.

\section{Non-linear regression technique}

This technique is based on an error minimization method where the error between the a priori known moments and the moments obtained after providing the raw voltage and the initial guess for the calibration coefficients are minimized. The first four moments used in this technique are defined as 


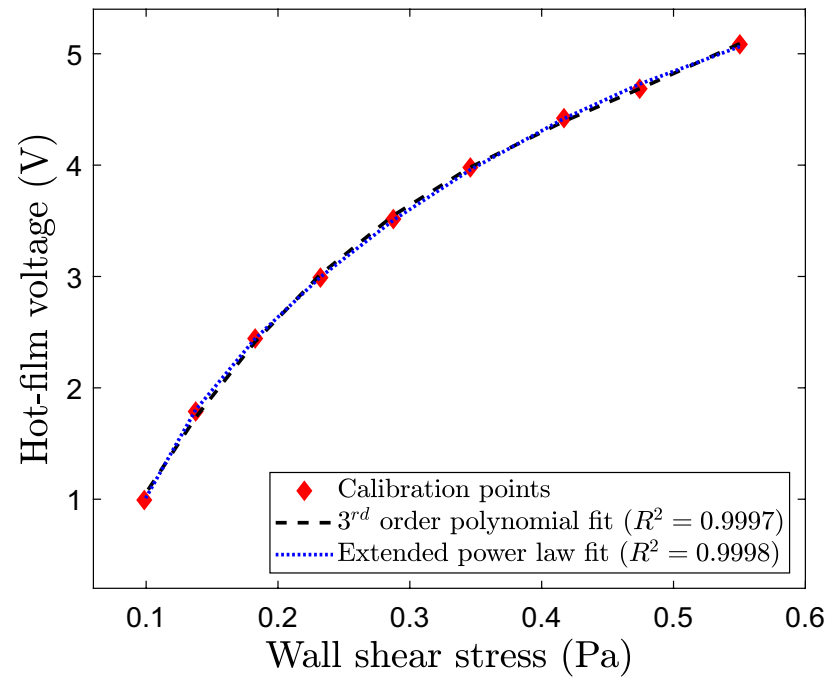

Fig. 1 Calibration plot of mean hot-film voltage against mean wallshear stress for $\operatorname{Re}_{\tau}=180$. The calibration curve is fit with a thirdorder polynomial and an extended power law. The ambient fluid temperature is maintained at $T=20.80^{\circ} \mathrm{C}$ with a precision of $\pm 0.01{ }^{\circ} \mathrm{C}$

follows: mean, $\mu(q)$, is the arithmetic average of the quantity $q$ (' $u$ ' for velocity and ' $\tau_{\mathrm{w}}$ ' for wall-shear stress), RMS, $\sigma\left(q^{\prime}\right)$, is the root mean square of the fluctuation about the mean $q^{\prime}=q-\mu(q)$, and skewness and flatness are given by $S\left(q^{\prime}\right)=\mu\left(q^{\prime 3}\right) / \sigma\left(q^{\prime}\right)^{3}$ and $F\left(q^{\prime}\right)=\mu\left(q^{\prime 4}\right) / \sigma\left(q^{\prime}\right)^{4}$, respectively. The function to be minimized is the sum of squares of relative errors in the first four moments, as shown in the following equation:

$E=\left(1-\frac{\mu_{r}}{\mu_{t}}\right)^{2}+\left(1-\frac{\sigma_{r}}{\sigma_{t}}\right)^{2}+\left(1-\frac{S_{r}}{S_{t}}\right)^{2}+\left(1-\frac{F_{r}}{F_{t}}\right)^{2}$.

Subscript ' $t$ ' shows the "true" moments which simply denotes the known moments. Subscript ' $r$ ' shows the moments calculated using regression. Two different functional forms of calibration, i.e., third-order polynomial and extended power law, are investigated in this study. The inputs for this regression technique are the raw voltage from the anemometer, "true" moments, and the initial guess for the calibration coefficients. To calculate the moments, the coefficients are input as variables and the time history of velocity or wall-shear stress and its first four moments are calculated. Moments are substituted into Eq. (1) and the error function $(E)$ is calculated. First, the error is calculated based on the initial guess for the coefficients given by the user. If the error is more than the tolerance threshold, then a new set of coefficients are calculated using non-linear regression. In the present study, MATLAB's fmincon, a non-linear programming solver, is used to calculate the new values of the calibration coefficients using the default interior point algorithm. This is an iterative algorithm where the non-linear regression

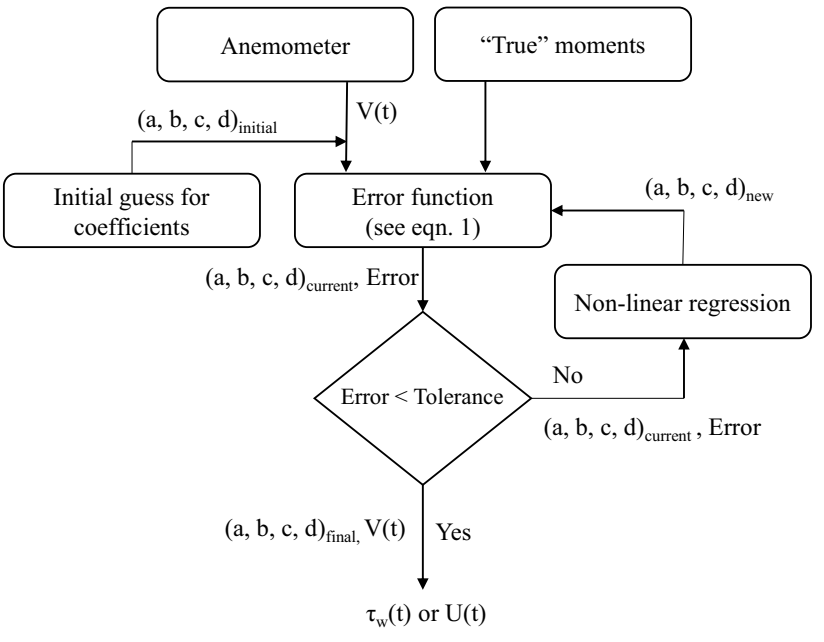

Fig. 2 A flowchart showing the various steps of this non-linear regression technique

keeps iterating until the error is below the tolerance threshold. Further details about this algorithm can be found in Dennis and Schnabel (1996). Once the error is below the tolerance threshold, the obtained (final) calibration coefficients are used to generate the regressed time history of velocity or wall-shear stress. A simple step-by-step flowchart of the method is shown in Fig. 2. Good initial guesses for the coefficients and a low tolerance threshold for the minimization, as with all other optimization techniques, are important parameters and need to be appropriately considered. We confirmed the validity of the presented technique using other standard minimization methods such as Solver in Excel, which produced very similar results.

\section{Sensitivity analysis}

To check the robustness of this technique, an exhaustive sensitivity analysis is conducted for $R e_{\tau}=180$ where the effect of type of calibration relationships, number of moments used for regression, and length of time series used are investigated. Later, in this section, the effect of Reynolds number on the presented technique is shown for two other Reynolds numbers $R e_{\tau}=61$ and 84 .

\subsection{Calibration relationships}

As stated before, the two commonly used calibration equations for thermal anemometry, third-order polynomial and extended power law, are used here. Table 1 shows a comparison of the moments obtained from the calibration and using the regression for wall-shear stress measurements at $R e_{\tau}=180$. It can be observed that there is an excellent agreement between the moments obtained from 
Table 1 Comparison of the first six moments obtained using calibration and regression for $R e_{\tau}=180$

\begin{tabular}{lccl}
\hline Moments & $\begin{array}{l}\text { Calibration } \\
\text { (third-order } \\
\text { polynomial) }\end{array}$ & $\begin{array}{l}\text { Regression } \\
\text { (third-order } \\
\text { polynomial) }\end{array}$ & $\begin{array}{l}\text { Regression } \\
\text { (extended power } \\
\text { law) }\end{array}$ \\
\hline$\mu\left(\tau_{\mathrm{w}}\right)(\mathrm{Pa})$ & 0.2536 & 0.2536 & 0.2536 \\
$\sigma\left(\tau_{\mathrm{w}}^{\prime}\right) / \mu\left(\tau_{\mathrm{w}}\right)$ & 0.2677 & 0.2677 & 0.2653 \\
$S\left(\tau_{\mathrm{w}}^{\prime}\right)$ & 0.5279 & 0.5279 & 0.5279 \\
$F\left(\tau_{\mathrm{w}}^{\prime}\right)$ & 2.8958 & 2.8956 & 2.8958 \\
$\mu\left(\tau_{\mathrm{w}}^{\prime 5}\right) / \sigma\left(\tau_{\mathrm{w}}^{\prime}\right)^{5}$ & 4.3040 & 4.3036 & 4.3036 \\
$\mu\left(\tau_{\mathrm{w}}^{\prime}\right) / \sigma\left(\tau_{\mathrm{w}}^{\prime}\right)^{6}$ & 15.2829 & 15.2793 & 15.4870 \\
\hline
\end{tabular}

calibration and non-linear regression using two different functional forms which confirms that this technique performs well in recovering the "true" moments. Fifth and sixth order moments, which are given by $\mu\left(\tau_{\mathrm{w}}^{\prime 5}\right) / \sigma\left(\tau_{\mathrm{w}}^{\prime}\right)^{5}$ and $\mu\left(\tau_{\mathrm{w}}^{\prime}{ }^{6}\right) / \sigma\left(\tau_{\mathrm{w}}^{\prime}\right)^{6}$, are also calculated, and it is observed that there is a good agreement in the moments obtained from regression and calibration till the sixth order. Figure 3 shows that there is a good match between the instantaneous normalized wall-shear stress fluctuations and their corresponding probability density functions (PDFs) obtained using calibration and regression for both calibration relationships where $t$ and $U_{b}$ represents time and bulk velocity, respectively. The PDF of wall-shear stress fluctuations obtained by Sreenivasan and Antonia (1977) using hot film in a channel flow facility at $R e_{\tau}=289$ is also shown for comparison.

\subsection{Number of moments used for regression}

Sensitivity of the non-linear regression to the number of moments used in Eq. (1) is investigated at $R e_{\tau}=180$ for the third-order polynomial fit. Regressions are conducted for the cases where errors in the first four, first three, and first two moments are minimized. Figure $4 a, b$ reveals good agreement between the PDFs and time series for the case where the first four and first three moments are employed for the non-linear regression, but if we focus on the peaks of the PDFs (shown in the inset plot of Fig. 4a), it is clear that the first four moments provide more accurate recovery of the PDF. The standard deviation of the error in the predicted time series for the first three moments is found to be $\sim 10^{-4}$, but, for the first four moments, it is $\sim 10^{-6}$. Thus, it can be said that both the first three and first four moments work well for the non-linear regression, but, for more accurate results, the first four moments should be used.

\subsection{Number of samples used for regression}

In the present study, the number of samples is converted to the corresponding non-dimensional convective time units.

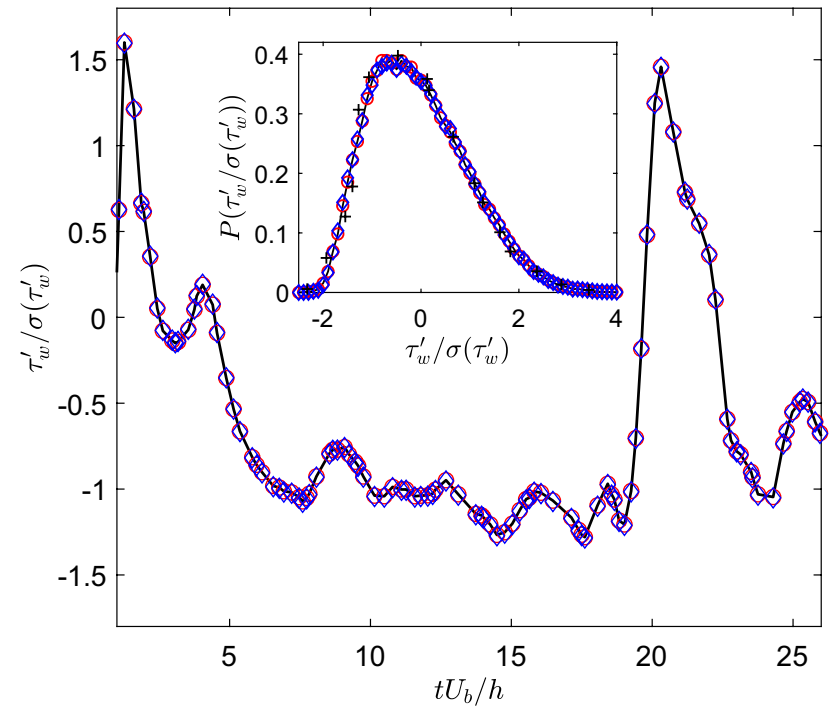

Fig. 3 Instantaneous normalized wall-shear stress fluctuations obtained using a hot film at $R e_{\tau}=180$ where the black line, red circles, and blue diamonds show the time history obtained using calibration, non-linear regression for a polynomial fit and non-linear regression for an extended power law fit, respectively. Data shown here are reduced by a factor of 5 for clarity. Inset plot shows PDFs of normalized wall-shear stress fluctuations at $R e_{\tau}=180$ where the line and symbols are as the main plot. Black plus represents PDF data obtained from Sreenivasan and Antonia (1977) for $R e_{\tau}=289$

Before employing the non-linear regression technique, it is necessary to check the statistical convergence of the first four moments which will be used for the regression, as this technique heavily depends on the first four moments. To do this, the entire time series is broken into smaller time segments for various convective times ranging from $t U_{\mathrm{b}} / h=1$ to 40,000 , where one convective time unit $\left(t U_{\mathrm{b}} / h=1\right)$ comprises only 18 data points. The first four moments are calculated for each time interval along the entire length of the time series and then the absolute percentage error is calculated based on reference moments for $t U_{\mathrm{b}} / h=40,000$, and the absolute percentage errors are then averaged. Figure 5a shows the variation of average absolute percentage errors of the first four moments obtained using calibration for different length of time series. It can be seen that, after 10,000 convective time units, all the four moments are converged within $5 \%$ of the moments obtained from the data comprising 40,000 convective time units. Thus, the data presented here can be considered statistically converged for $t U_{\mathrm{b}} / h>10,000$. A related, but distinct question is what is the minimum length of time series required for the regression technique to work? To answer this, the entire time series is again broken into smaller time segments, for various convective times ranging from $t U_{\mathrm{b}} / h=1$ to 40,000 . The first four moments are calculated for each of the time intervals along the entire length of the time series, and then, these 

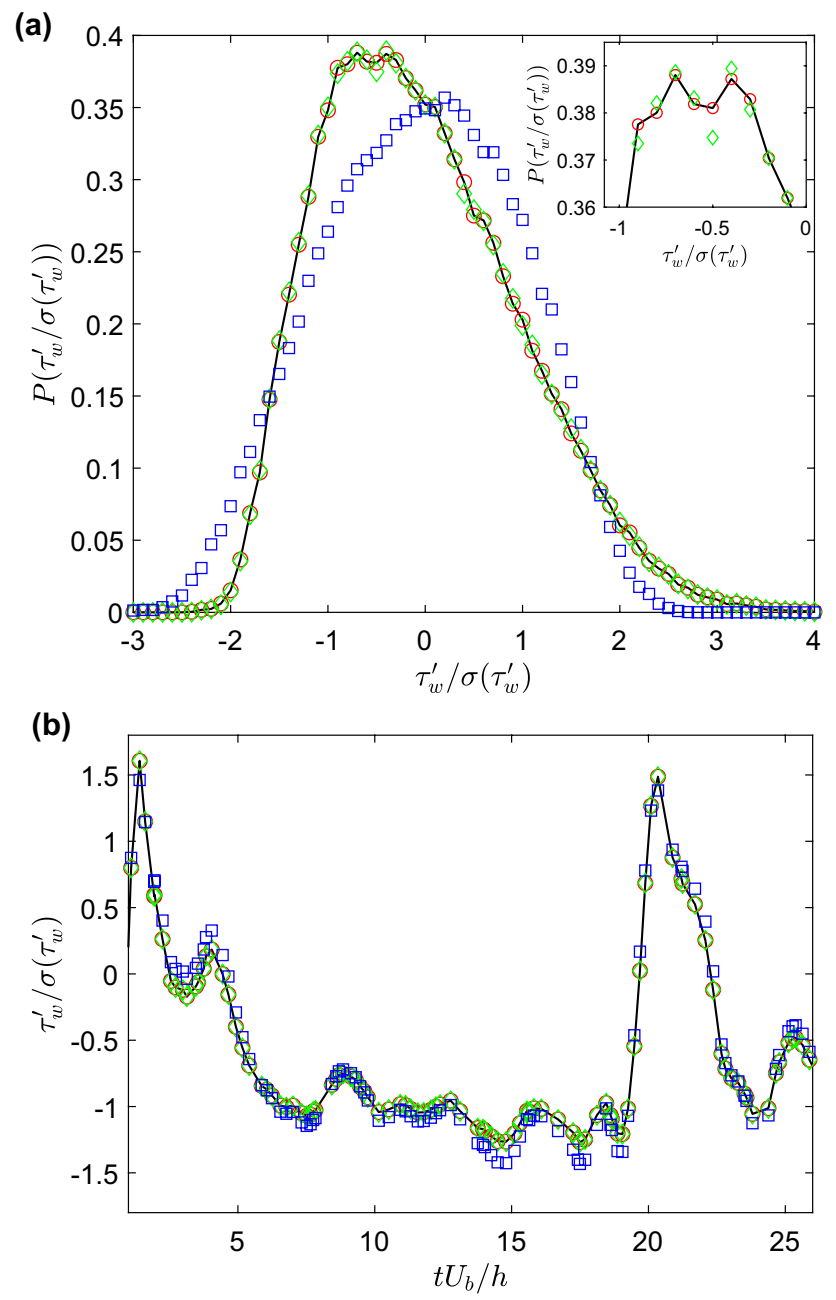

Fig. 4 a PDFs of normalized wall-shear stress fluctuations obtained using a hot film at $R e_{\tau}=180$ where non-linear regression is used for a third-order polynomial fit. Black line shows the PDF obtained using calibration, and the red circles, green diamonds, and blue squares show the PDFs obtained when the first four, first three, and first two moments are used for the minimization of Eq. (1), respectively. b Instantaneous wall-shear stress fluctuations where the line and symbols are as in Fig. 4a. Data shown here are reduced by a factor of 5 for clarity

moments are used for the regression over the same length of time series. The absolute percentage error is calculated between the calibrated and regressed moments, and the absolute percentage errors are then averaged. Figure $5 \mathrm{~b}$ shows the average absolute percentage error in the regressed moments for various lengths of time series. It is observed that the regression works well in recovering the moments for the length of time series above $t U_{\mathrm{b}} / h=100$. Therefore, making a decision regarding the length of time series necessary should depend mainly on whether the moments are converged; however, it can be seen that the non-linear regression technique is quite robust and capable of recovering

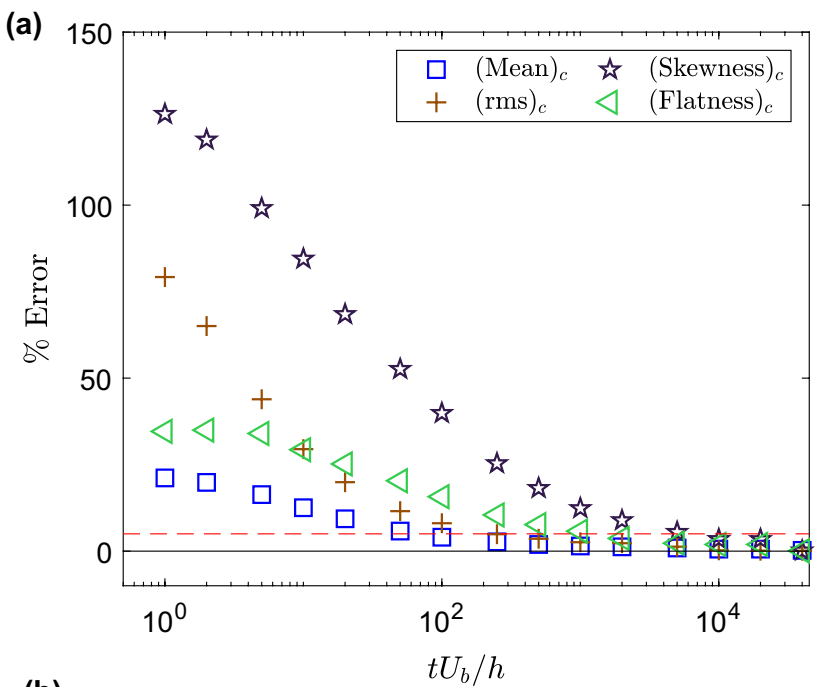

(b)

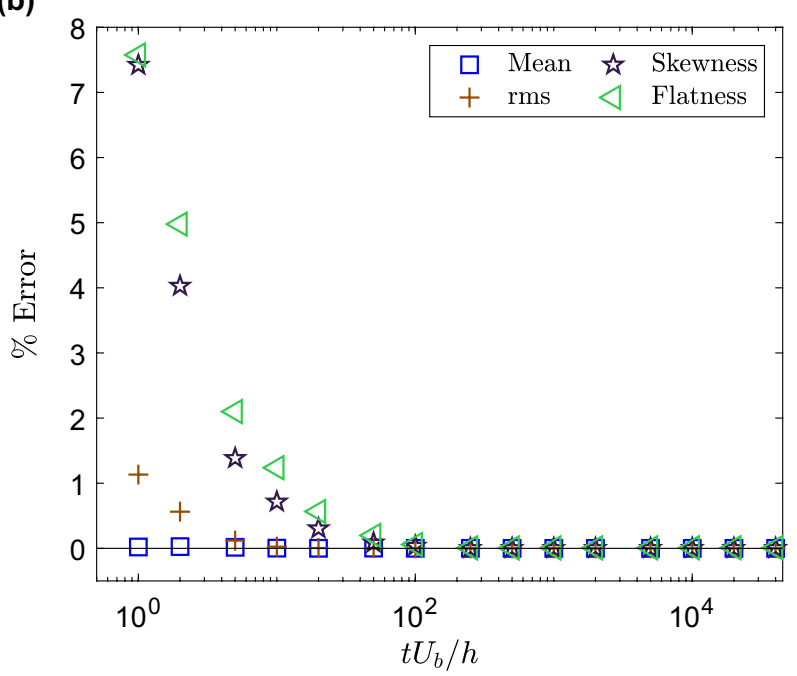

Fig. 5 a Variation of the average absolute \% error for the first four moments obtained using calibration for $R e_{\tau}=180$ with the convective time over which the measurement is conducted, where the error is calculated using the corresponding moments obtained for highest convective time $\left(t U_{\mathrm{b}} / h=40,000\right)$. Red dashed line and black solid line shows the constant values of $5 \%$ and $0 \%$, respectively. b Variation of the average absolute $\%$ error in the first four moments obtained using calibration and regression for various lengths of measurement times. Black solid line (-) shows the constant value of $0 \%$

the 'input' moments even for as small a sample size as $t U_{\mathrm{b}} / h=100$.

\subsection{Reynolds number effects}

The effect of Reynolds number on the regression for wallshear stress measurements in the channel flow is investigated by following a similar approach for two other Reynolds numbers $R e_{\tau}=61$ and 84 . Figure 6 shows the instantaneous wall-shear stress fluctuations and the corresponding PDFs obtained using calibration and regression for $R e_{\tau}=61$ and 
84. There is observed to be an excellent agreement for both of these Reynolds numbers which confirms the validity of the presented technique for wall-shear stress data obtained in different flow conditions at least over this range of Reynolds number.

\section{Validation against hot-wire data}

An additional study is made to check how well this technique works for an independent test case where a hot wire is employed for instantaneous velocity measurements at higher Reynolds numbers. Data of streamwise velocity measurements obtained with a hot wire from a channel flow facility at the University of Melbourne for $R e_{\tau}=1053$, reported in $\mathrm{Ng}$ et al. (2011), are used. Here, the "true" moments were

(a)
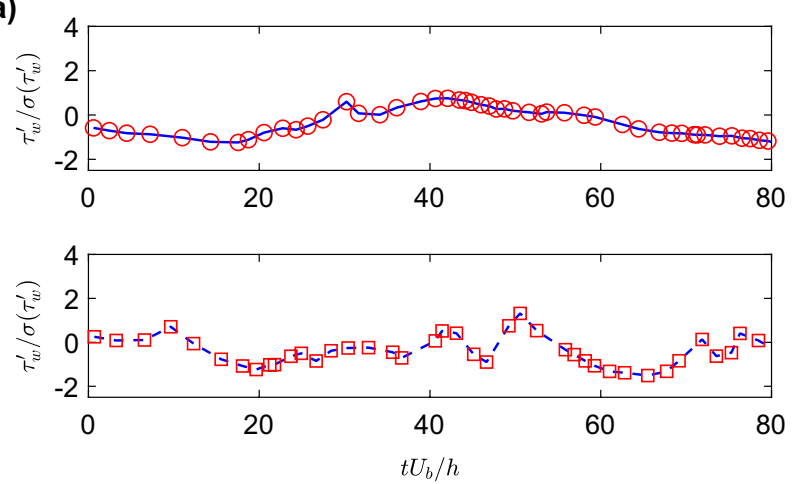

(b)

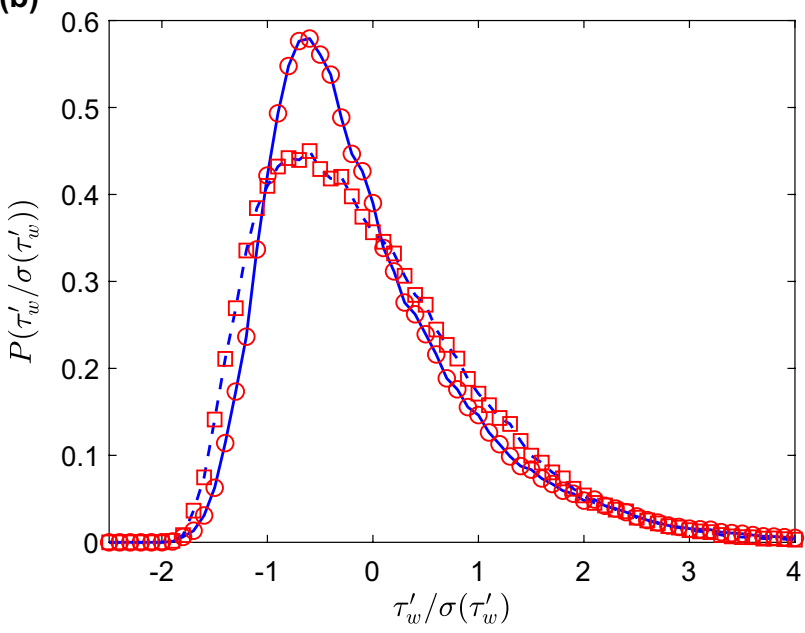

Fig. 6 a (Top to bottom) Instantaneous normalized wall-shear stress fluctuations obtained using a hot film at $R e_{\tau}=61$ and 84 . Blue solid and dashed lines show the data obtained from in situ calibration for $R e_{\tau}=61$ and 84, respectively. Red circles and red squares indicate the regression data obtained using third-order polynomial calibration form for $R e_{\tau}=61$ and 84, respectively. Data shown here are reduced by a factor of 12 for clarity. b PDF of normalized wall-shear stress fluctuations where various lines and symbols represent the same quantities as in $\mathbf{a}$ obtained from the velocity signals measured using a hot-wire probe that was calibrated in situ at the channel centerline against the centerline velocity measured by a Pitot-static probe. The spanwise width of hot-wire probe was $l^{+}=22$ with further details regarding the experimental procedure found in $\mathrm{Ng}$ et al. (2011). Data of velocities for three wall normal locations at $y^{+}=16,107$ and 1053 for $R e_{\tau}=1053$ are investigated.

The procedure used for the regression is briefly explained here. Data were a long-time history of raw hot-wire voltage and the corresponding calibrated velocity data for the three wall normal distances at $R e_{\tau}=1053$. We first calculated the first four moments from the calibrated velocity signal and then used these moments for the regression. A thirdorder polynomial calibration relationship was assumed as the fitting function. After running the regression, the predicted calibration coefficients were obtained. These coefficients were then used to reconstruct velocity time series from the hot-wire data. Table 2 shows an excellent agreement of moments obtained from calibration and non-linear regression for $R e_{\tau}=1053$ at $y^{+}=107$ which further validates this technique in recovering the moments. Segments of the instantaneous normalized streamwise velocity fluctuations and their corresponding PDFs shown in Fig. 7a, $\mathrm{b}$, respectively, show that this technique performs well in reconstructing the signal for all three wall normal locations where $U_{\mathrm{cl}}$ represents centerline velocity. Validation against the hot-wire data shows that this technique works for different kinds of flow conditions as the hot-film and hot-wire data shown here have different skewness, i.e., $S\left(\tau_{\mathrm{w}}^{\prime}\right)>0$ for hot film at $R e_{\tau}=180$, but $S\left(u^{\prime}\right)<0$ for hot-wire at $R e_{\tau}=1053$ at $y^{+}=107$. As we have shown that the non-linear regression technique performs well for hot-film and hot-wire data collected in fully developed channel flow for friction Reynolds numbers between $61 \leq R e_{\tau} \leq 1053$, we expect that the non-linear regression technique should continue to perform well at even higher Reynolds numbers as a method for minimizing (re-)calibrations and correcting non-thermal drift. However, we note that this technique is dependent on the quality of the inputs and cannot account for, or correct, measurement resolution issues necessarily encountered at high Reynolds numbers (see, for example, Hutchins et al. 2009).

\section{Potential scenarios for application}

In this section, four potential scenarios for application are discussed where the proposed technique might be useful in practical experiments. 

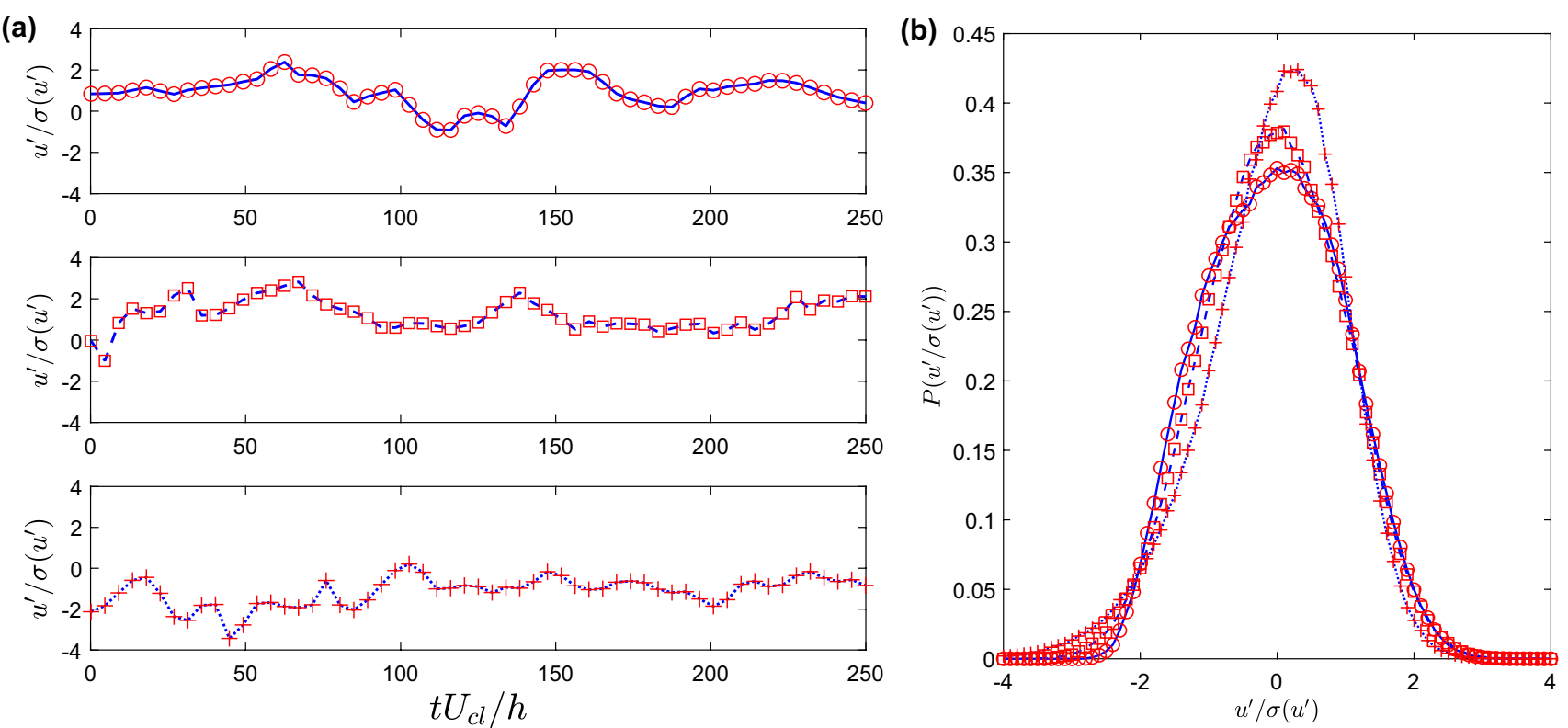

Fig. 7 a (Top to bottom) Instantaneous normalized streamwise velocity fluctuations obtained using a hot wire at $R e_{\tau}=1053$ for $y^{+}=16$, 107, and 1053. Blue solid, dashed, and dotted lines show the data obtained from in situ calibration for $y^{+}=16,107$, and 1053, respectively. Red circles, red squares, and red pluses indicates the regres-

Table 2 Comparison of the first four moments obtained using calibration and regression for $R e_{\tau}=1053$ at $y^{+}=107$

\begin{tabular}{lcc}
\hline & $\begin{array}{l}\text { Calibration (third-order } \\
\text { polynomial) }\end{array}$ & $\begin{array}{c}\text { Regression (third- } \\
\text { order polynomial) }\end{array}$ \\
\hline$\mu(U)(\mathrm{m} / \mathrm{s})$ & 5.3926 & 5.3926 \\
$\sigma\left(u^{\prime}\right) / \mu(U)$ & 0.1171 & 0.1171 \\
$S\left(u^{\prime}\right)$ & -0.0481 & -0.0481 \\
$F\left(u^{\prime}\right)$ & 2.6919 & 2.6933 \\
\hline
\end{tabular}

\subsection{Non-thermal drift in long runs}

In this scenario, use of the presented technique for correcting the data which have suffered non-thermal drifts is investigated. There are two types of cases which are possible in case of non-thermal drifts: drift from a sensor when simultaneous multi-probe measurements are conducted in the fully developed region of the flow or drift from the same hot-wire/ hot-film sensor during a long measurement. In the former case, since the measurements are conducted in the fully developed region, the long-time statistics should be the same for all the sensors. In this section, the non-thermally drifted data (most likely due to contaminant deposition) from a hotfilm sensor are corrected using the moments acquired from another hot film, when both sensors were running simultaneously in the fully developed region of the flow. Longrun measurements of wall-shear stress were conducted for sion data obtained using third-order polynomial calibration form for $R e_{\tau}=1053$ at $y^{+}=16,107$, and 1053, respectively. Data shown here are reduced by a factor of 30 for clarity. b PDF of normalized streamwise velocity fluctuations at $R e_{\tau}=1053$ where various lines and symbols represent the same quantities as in $\mathbf{a}$

$R e_{\tau}=84$ in the Liverpool channel flow facility using two glue-on hot films. Figure 8a shows the test section with the location of the two hot films. Both hot films are located fivechannel half-heights away from the side wall, but hot film 1 and hot film 2 are located 496 and 491 channel half-heights away from the inlet, respectively. The long-time moments of the wall-shear stress should be the same at these two spatial locations. Simultaneous acquisition of data from both hot films is conducted at a typical data rate of around $250 \mathrm{~Hz}$. Pre- and post-calibration is conducted with a long-run measurement of about $t U_{\mathrm{b}} / h=10,000$. An isothermal condition was maintained for the entire run where the fluid temperature varied only by about $\pm 0.01^{\circ} \mathrm{C}$. Hot-film 2 is observed to have drifted in the voltage output over time which is an example of non-thermal drift. Figure $8 \mathrm{~b}$ shows a pictorial representation of the current scenario. As the drift led to a non-stationary mean voltage value, a first-order polynominal was fitted to the data and used to remove the linear trend in the drifted data, and then, the regression technique was conducted using moments from hot film 1 . Figure $8 \mathrm{c}$ shows the time history of the wall-shear stress obtained from the entire run. It can be observed that hot film 2 has suffered calibration drift prior to the removal of the aforementioned linear trend. From Fig. 8d it can be said that the non-linear regression works well in recovering the PDF of wall-shear stress from the hot film which has suffered non-thermal drifts. The standard deviation of the error in the two PDFs is found to be about 0.0048 . 


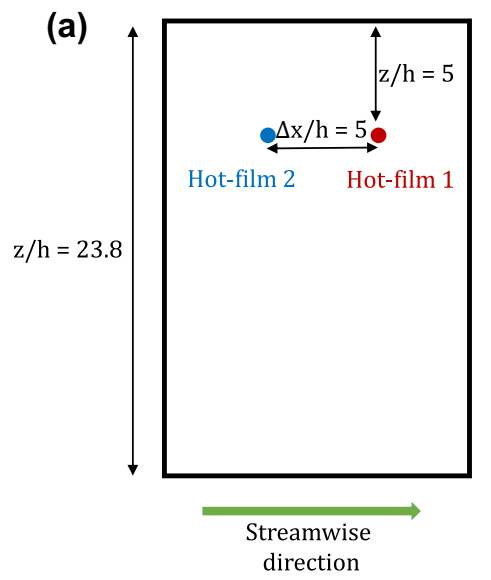

(b)

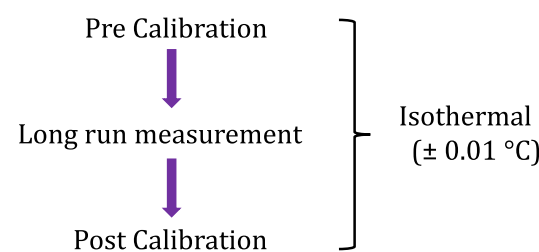

Hot film 1: Pre Calibration $=$ Post Calibration (No drift)

Hot film 2: Pre Calibration $\neq$ Post Calibration (Non-thermal drift)
Use moments obtained from hot-film 1 to conduct non-linear regression on voltage data from hot-film 2 (c)
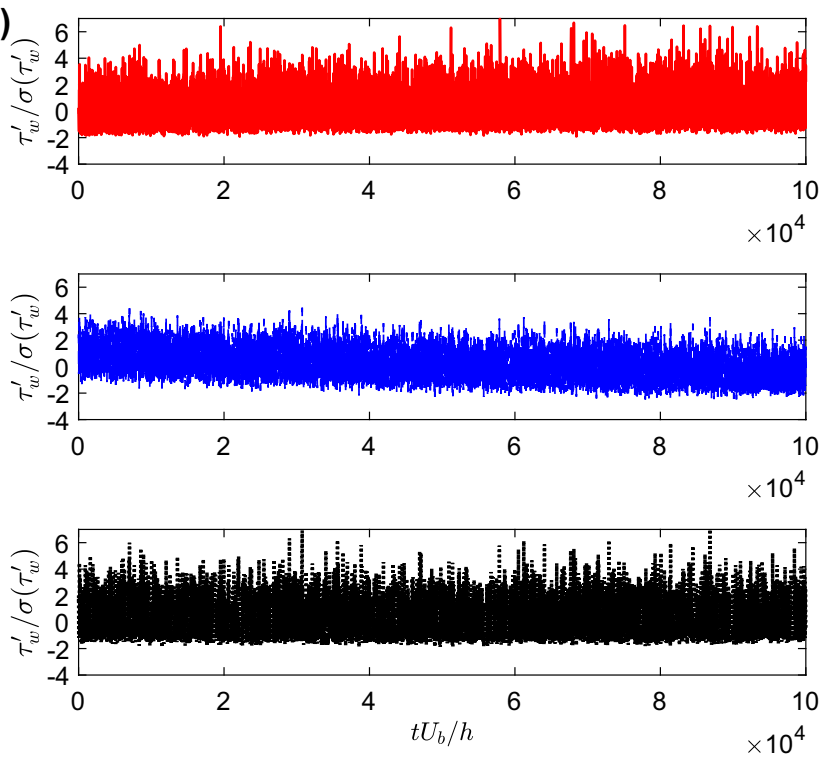

(d)

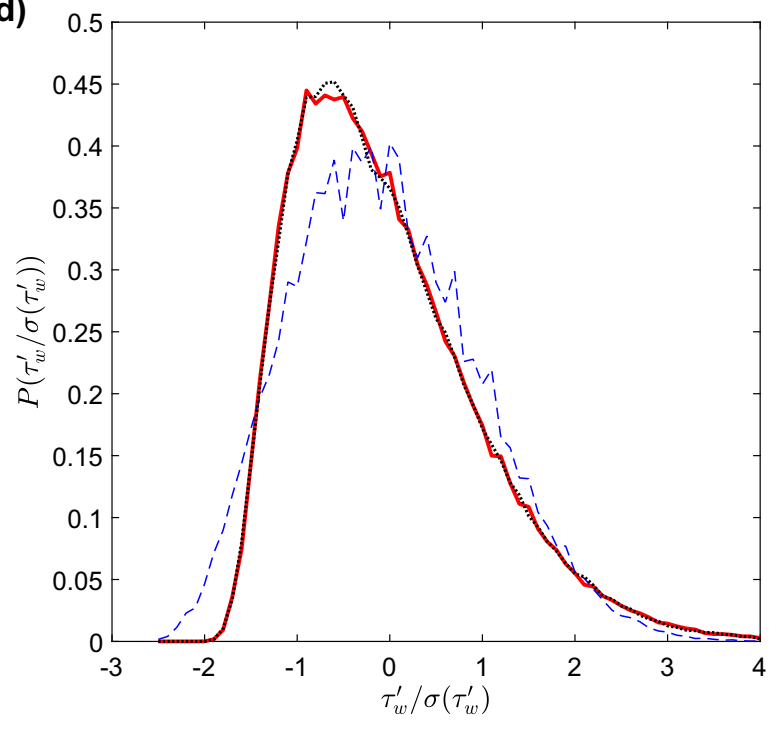

Fig. 8 a Schematic of the test section of the channel flow facility (not to scale); b procedure which is followed in the current scenario; c (top to bottom) Instantaneous wall-shear stress fluctuations for $R e_{\tau}=84$ obtained using hot film 1 (red line) and hot film 2 (blue

Correcting data obtained from a hot wire/hot film which has suffered non-thermal drifts are also particularly relevant for the cases where long measurements are required for studying conditional properties of the flow. In the absence of a second probe, this technique can provide an alternative to correct for non-thermal calibration drifts by taking the moments from the initial long-run measurement, before the non-thermal drift of the sensor becomes significant. Then, the obtained moments can be used to correct for the drifted data obtained later for the same flow conditions to reconstruct the velocity or wall-shear stress signal. A previous study by Talluru et al. (2014) has discussed a technique to correct for the drift issues in dashed line) and the corrected data from hot film 2 (black dotted line). d PDF of wall-shear stress fluctuations with the lines indicated as in $\mathbf{c}$

hot-wire anemometry which is applicable for all kinds of drifts (thermal or non-thermal). This technique relies on regular recalibrations of the hot wire in the free stream of a boundary layer during the experiment in what they term intermediate single-point recalibration (ISPR). Whilst our proposed technique can be useful in minimizing the number of recalibrations performed (in the case of nonthermal drifts), thus saving time, it can also be used on glue-on hot-film probes where the ISPR methodology may not be practical. Correction for humidity effects in air flow (another example of non-thermal drifts) was studied previously by Durst et al. (1996). Although we could not check the validity of our correction technique for humidity effects, because we used a liquid as our working fluid, it is 
believed that this technique can correct for the humidity effects if the first three or four moments can be acquired a priori for similar flow conditions, at lower humidity.

\subsection{Estimation from numerical database}

This non-linear regression technique can be useful in the scenario when a direct calibration is not possible, but the moments for that particular flow can be obtained independently from a numerical database. Using the present technique, an attempt is made here to reconstruct the wall-shear stress fluctuations from the hot-film voltage data using the moments obtained from the DNS database of Hu et al. (2006). The DNS data used are for $R e_{\tau}=180$ in a channel flow. Here, the size of the computation box is $L_{x} \times L_{y} \times L_{z}=24 \times 2 \times 12$ with grid points $N_{x} \times N_{y} \times N_{z}=256 \times 121 \times 256$ in the $x$ (streamwise), $y$ (wall normal) and $z$ (spanwise) directions. The streamwise and spanwise grid spacings are $x^{+}=16.88$ and $z^{+}=8.44$, respectively. Further description of the numerical method can be found in Hu et al. (2006). The moments of wallshear stress obtained from the present experiment and the DNS (and, thus, the shape of the corresponding PDF) are not equal, even at the same Reynolds number. This difference in the moments is generally attributed to the spatial and temporal resolution issues of the hot films and the substrate on which these hot films are glued (Khoo et al. 2001; Alfredsson et al. 1988). Therefore, one should be careful while taking moments from the numerical database and using it for this regression technique to estimate meaningful time history from the raw hot-film voltage data. The method suggested by Chin et al. (2009) can be a potential way to correct for the differences in the moments through spatial filtering of the DNS data to match the spatial resolution of the sensor. In the current scenario, regression is conducted using the thirdorder polynomial as the fitting function for the calibration equation where the input to the regression is the moments from the DNS and the measurement. From Fig. 9, it can be seen that the regression technique is able to provide a fairly good estimate of the time histories obtained using two different sets of moments. The inset plot of Fig. 9 shows that the PDF of the wall-shear stress fluctuations obtained by DNS is in fairly good agreement with the PDF obtained using DNS except at the peak, which highlights the aforementioned differences between physical experiments and numerical databases. It can be seen that although two different sets of moments are used, this regression technique performs well in providing qualitatively similar wall-shear stress signals from the same hot-film voltage data.

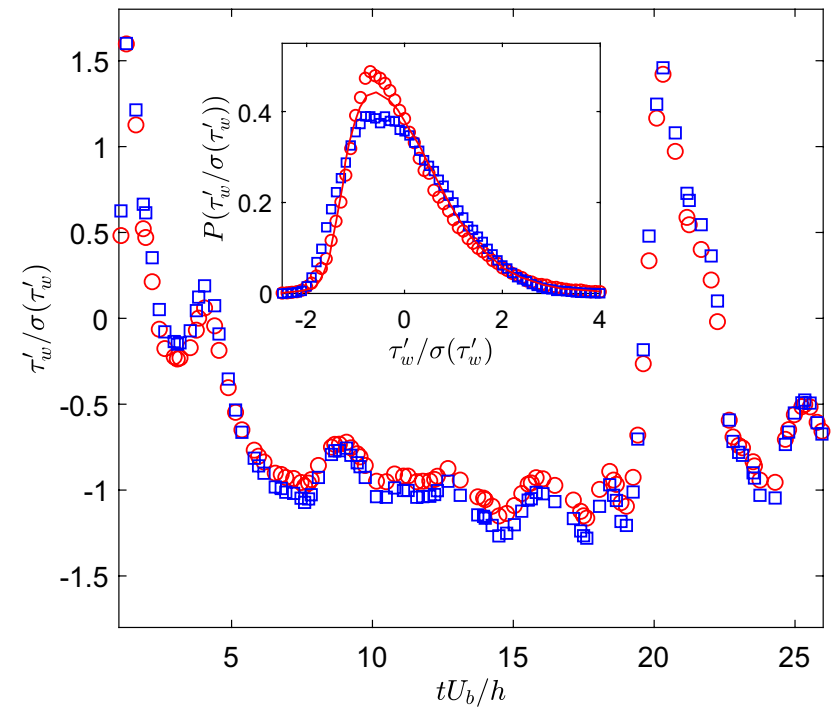

Fig. 9 Instantaneous normalized wall-shear stress fluctuations at $R e_{\tau}=180$ obtained using non-linear regression for a third-order polynomial fit where red circles and blue squares represent data when the moments were obtained from DNS (Hu et al. 2006) and the present experiment, respectively. Data shown here are reduced by a factor of 5 for clarity. Inset plot shows the PDFs of normalized wallshear stress fluctuations at $R e_{\tau}=180$ where symbols represent the same quantities as the main plot. Red line shows the PDF of normalized wall-shear stress fluctuations obtained using DNS at $\boldsymbol{R e}_{\tau}=180$ (Hu et al. 2006)

\subsection{Transferability to other Reynolds numbers}

Until now, the one condition for this technique to work is the availability of well-converged moments for the same Reynolds number. In this scenario, an attempt is made to investigate the robustness of this technique if the moments for Reynolds numbers close to the desired Reynolds number are available. It is tested to see if we can estimate the wallshear stress signals from the hot-film voltage data without conducting an in situ calibration, if the moments are available for a different Reynolds number. To make this technique work, the one condition is that, for every measurement run, there should be one set of measurements done at the same Reynolds number where the moments of wall-shear stress are already known. Therefore, using the new hot-film voltage signals and the previously known moments, both at the same Reynolds number, a new set of calibration coefficients can be obtained via regression. And then, this set of calibration coefficients can be used for the conversion of voltage to wallshear stress information for the other Reynolds number. This hypothesis is based on the assumption that the calibration curve obtained for a particular Reynolds number should also work well for the other Reynolds number.

To validate the accuracy of this hypothesis, an illustrative example is discussed here for the wall-shear stress measurements conducted in a channel flow facility. Two 
different sets of measurements are conducted on two different days where on day 1 a long-run measurement was conducted for $R e_{\tau}=84$ with a pre- and post-calibration, and on day 2, long-run measurements were conducted for $R e_{\tau}=61,73$, and 84 again with pre- and post-calibration, using the same hot-film sensor located at 496 and five channel half-heights away from the inlet and side wall, respectively. An isothermal condition was maintained with temperature variation of $\pm 0.01^{\circ} \mathrm{C}$ during the entire experimental run, thus, providing negligible differences in the pre- and post-calibration. Using the moments obtained on day 1 for $R e_{\tau}=84$, regression is conducted on the hot-film voltage signal obtained on day 2 for $R e_{\tau}=84$ to obtain a new set of calibration coefficients assuming a third-order polynomial calibration relationship. Figure 10a shows the comparison of the calibration curves obtained using regression for $R e_{\tau}=84$ and using in situ calibration on day 2. It can be observed that the regression provides a good estimate of the calibration curve except at the lower and higher ends of the curve. The calibration coefficients obtained via regression are used for the estimation of wall-shear stress signal for $R e_{\tau}=61$ and 73. Figure 10b, $\mathrm{c}$ shows the comparison of wall-shear stress fluctuations and their corresponding PDFs for $R e_{\tau}=61$ and 73. It can be observed that the calibration coefficients obtained using regression for $R e_{\tau}=84$ provide a fairly good estimate of the time history of wall-shear stress and the corresponding PDFs for $R e_{\tau}=73$. Figure $10 \mathrm{~b}$ shows a fairly good estimate of the wall-shear stress obtained for $R e_{\tau}=61$ using the two different aforementioned methods. However, Fig. 10c shows that, for $R e_{\tau}=61$, there is less good agreement in the PDFs of wall-shear stress obtained using the two different methods. The reason for this less good estimate is clear in the calibration curves of Fig. 10a. At $R e_{\tau}=73$, we are still within the range where the curves obtained from calibration and regression are very similar, and so the technique works well. At $R e_{\tau}=61$, there is a small difference in the curves, and hence, there is an error in the estimated wall-shear stress using the regression technique. Of course, the wall-shear stress values indicated for each $R e_{\tau}$ on Fig. 10a are the average values and the fluctuations in the flow mean that a range of the calibration curve is being utilized at each Reynolds number. As $R e_{\tau}=61$ which is at the lower end of this curve, it is evident that high wall-shear stress events will likely be recovered well, (because they will be in a region of the calibration which is still valid). However, low wall-shear stress events will be taking the calibration to the extremity of its validity and, therefore, producing less accurate results. This interpretation is clearly evident in the PDF for $R e_{\tau}=61$ in Fig. 10c (and also to some extent in the time series of Fig. 10b), where, at the low wall-shear stress end of the PDF, we see significant differences in the
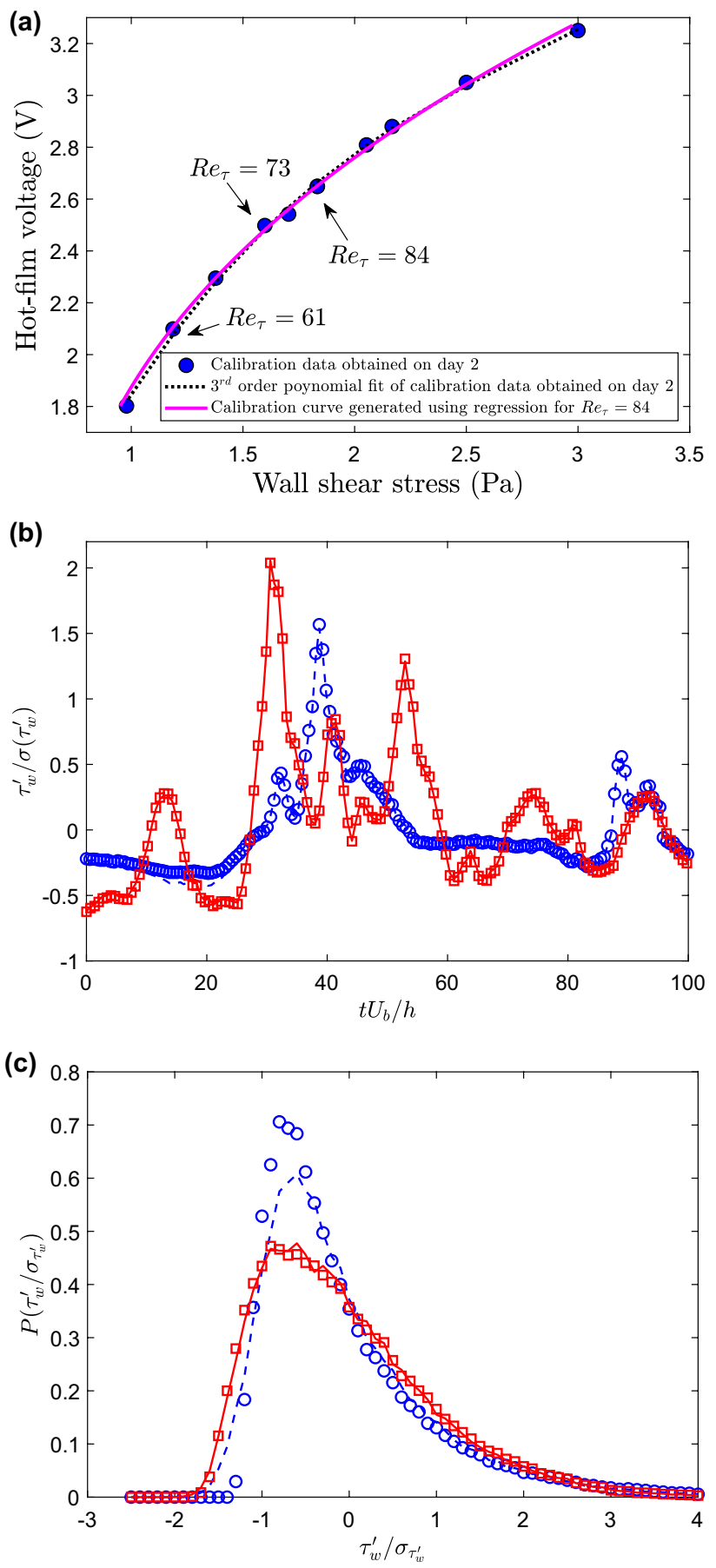

Fig. 10 a Calibration plot of mean hot-film voltage against mean wall-shear stress; $\mathbf{b}$ instantaneous normalized wall-shear stress fluctuations obtained using a hot film at $R e_{\tau}=61$ shown by blue dashed line and blue circles for calibration equation obtained using calibration and regression based on moments for $R e_{\tau}=84$, respectively. Instantaneous normalized wall-shear stress fluctuations obtained using a hot film at $R e_{\tau}=73$ shown by red dashed line and red squares for calibration equation obtained using calibration and regression based on moments for $R e_{\tau}=84$, respectively. Data shown here are reduced by a factor of 5 for clarity. $\mathbf{c}$ PDFs of normalized wall-shear stress fluctuations where the line and symbols are as $\mathbf{b}$ 
curves from regression and calibration, whereas, at the high wall-shear stress end, the agreement appears to be much better. We conclude that this technique works well if the wall-shear stress at the new $R e_{\tau}$ is largely within the range of the reference $R e_{\tau}$. This is to be expected and is true for any calibration technique (i.e., the experiment has to be conducted in the range that the calibration is valid).

\subsection{Wall-shear stress measurements in external flows}

Our proposed technique can also have applications in external flows where the mean wall-shear stress is not a simple function of the mean pressure drop, e.g., zero-pressure gradient turbulent boundary layers. Although there are "direct" measures of mean wall-shear stress such as the floating element drag balance and oil film interferometry (see, for example, the review of Fernholz et al. 1996), the Clauser chart probably remains the most commonly used method to calibrate hot films, which adds uncertainty in the measurement (Hutchins et al. 2011). In these kinds of flows, or in more complicated external flows, e.g., flow with strong adverse pressure gradient where the Clauser chart and Preston tubes do not work, the presented technique can be of potential use if it is possible to reach the viscous sublayer using an independent measurement technique such as laser doppler velocimetry (LDV) or near-wall hot wire probes at various spatial locations in the flow. Once the moments are known from the velocity gradient data, wall-shear stress data can be recovered from the hot-film raw voltage signal using the non-linear regression. If we want to study the spatial distribution of wall-shear stress using simultaneous measurements of multi sensor probes, the proposed technique can be particularly useful, because, then, a single LDV or other probing devices can be used to measure the moments at various spatial locations, and later, using multiple hot-film probes, simultaneous measurements can be conducted on those spatial locations.

\section{Conclusion}

In this paper, a novel non-linear regression technique to minimize the recalibration in thermal anemometry is investigated. The proposed technique works well in predicting the calibration coefficients for velocity and wall-shear stress data obtained using thermal anemometry measurements. Robustness of this technique has been checked against two commonly used calibration equations and using data acquired from both hot-wire and glue-on hot-film probes. Sensitivity of the non-linear regression to the number of included moments indicates that the first three moments provide a reasonable approximation, but that use of the first four moments provides better agreement. One strength of the technique is that it can account for non-thermal calibration drifts, thereby potentially reducing the need for continuous in situ post-calibration throughout a long measurement. When the moments of wall-shear stress are obtained from a numerical database, we have demonstrated that the non-linear regression technique reported here can provide a reasonable estimate of the wall-shear stress signal. This technique is also shown to work well in the estimation of wall-shear stress from the hotfilm voltage signal, if the moments of the wall-shear stress at a nearby Reynolds number are available from a previous measurement. Finally, this technique can also potentially be used to recover the time series history of wall-shear stress when direct calibration methods are not readily available, such as in many external flows.

Acknowledgements This work has been supported by the Air Force Office of Scientific Research through Grant FA9550-16-1-0076. We would also like to thank Professor Ivan Marusic for access to the Melbourne data reported in $\mathrm{Ng}$ et al. (2011).

Open Access This article is distributed under the terms of the Creative Commons Attribution 4.0 International License (http://creativeco mmons.org/licenses/by/4.0/), which permits unrestricted use, distribution, and reproduction in any medium, provided you give appropriate credit to the original author(s) and the source, provide a link to the Creative Commons license, and indicate if changes were made.

\section{References}

Alfredsson PH, Johansson AV, Haritonidis JH, Eckelmann H (1988) The fluctuating wall-shear stress and the velocity field in the viscous sublayer. Phys Fluids 31(5):1026-1033. https://doi. org/10.1063/1.866783

Bruun HH (1995) Hot-wire anemometry: principles and signal analysis, 1st edn. Oxford University Press, New York

Chin CC, Hutchins N, Ooi ASH, Marusic I (2009) Use of DNS data to investigate spatial resolution issues in measurements of wall bounded turbulence. Meas Sci Technol 20(11):115401

Cimbala JM, Park WJ (1990) A direct hot-wire calibration technique to account for ambient temperature drift in incompressible flow. Exp Fluids 8(5):299-300. https://doi.org/10.1007/BF00187234

Collis DC (1954) The dust problem in hot-wire anemometry. Aeronaut Quart 4(1):93-102. https://doi.org/10.1017/S0001925900000810

Comte-Bellot G (1976) Hot-wire anemometry. Ann Rev Fluid Mech 8(1):209-231. https://doi.org/10.1146/annurev.f1.08.01017 6.001233

Dennis JE Jr, Schnabel RB (1996) Numerical methods for unconstrained optimization and nonlinear equations (classics in applied mathematics), vol 16. Society for Industrial and Applied Mathematics, Philadelphia

Durst F, Noppenberger S, Still M, Venzke H (1996) Influence of humidity on hot-wire measurements. Meas Sci Technol 7(10):15171528. https://doi.org/10.1088/0957-0233/7/10/021

Fernholz HH, Janke G, Schober M, Wagner PM, Warnack D (1996) New developments and applications of skin-friction measuring techniques. Meas Sci Technol 7:1396-1409 
Hu Z, Morfey CL, Sandham ND (2006) Wall pressure and shear stress spectra from direct simulations of channel flow. AIAA J 44(7):1541-1549. https://doi.org/10.2514/1.17638

Hultmark M, Smits AJ (2010) Temperature corrections for constant temperature and constant current hot-wire anemometers. Meas Sci Technol 21(10):105404. https://doi.org/10.1088/09570233/21/10/105404

Hutchins N, Nickels T, Marusic I, Chong M (2009) Hot-wire spatial resolution issues in wall-bounded turbulence. J Fluid Mech 635:103-136

Hutchins N, Monty J, Ganapathisubramani B, Ng H, Marusic I (2011) Three-dimensional conditional structure of a high Reynolds number turbulent boundary layer. J Fluid Mech 673:255-285

Khoo BC, Chew YT, Teo CJ (2001) Near-wall hot-wire measurements. Exp Fluids 5:494-505. https://doi.org/10.1007/s003480100304

Ligrani P, Bradshaw P (1987) Spatial resolution and measurement of turbulence in the viscous sublayer using subminiature hot-wire probes. Exp Fluids 5:407-417

Ng HCH, Monty JP, Hutchins N, Chong MS, Marusic I (2011) Comparison of turbulent channel and pipe flows with varying Reynolds number. Exp Fluids 51:1261-1281. https://doi.org/10.1007/s0034 8-011-1143-X

Sreenivasan KR, Antonia RA (1977) Properties of wall shear stress fluctuations in a turbulent duct flow. J Appl Mech 44(3):389-395. https://doi.org/10.1115/1.3424089
Talluru KM, Kulandaivelu V, Hutchins N, Marusic I (2014) A calibration technique to correct sensor drift issues in hot-wire anemometry. Meas Sci Technol 25(10):105304. https://doi. org/10.1088/0957-0233/25/10/105304

Tropea C, Yarin AL, Foss JF (2007) Springer handbook of experimental fluid mechanics. Springer-Verlag, Berlin

Whalley RD, Park JS, Kushwaha A, Dennis DJC, Graham MD, Poole RJ (2017) Low-drag events in transitional wall-bounded turbulence. Phys Rev Fluids 2:034602. https://doi.org/10.1103/PhysR evFluids.2.034602

Wu S, Bose N (1994) An extended power law model for the calibration of hot-wire/hot-film constant temperature probes. Int J Heat Mass Transf 37(3):437-442. https://doi.org/10.1016/00179310(94)90077-9

Publisher's Note Springer Nature remains neutral with regard to jurisdictional claims in published maps and institutional affiliations. 